\title{
Sicher verpackt
}

\section{Verblisterung in der ambulanten Pflege}

Arzneimitteltherapiesicherheit (AMTS) und Medikationsmanagement sind derzeit wichtige Begriffe in der Patientenversorgung. Die zunehmende Lebenserwartung unserer Gesellschaft, hat auch eine erhöhte Multimorbidität und Polypharmazie zur Folge.

Schon seit einigen Jahren wird diskutiert, ob ein gemeinsam von Arzt/Pflegeeinrichtung/Apotheke gepflegter Medikationsplan zusammen mit einer (Neu-)Verblisterung der verordneten Arzneien die AMTS in Heimen fördern kann. Mit zunehmendem Trend, Patienten möglichst lange in ihrer häuslichen Umgebung zu belassen, stellt sich inzwischen auch die Frage, ob nicht ebenso für ambulante Pflegedienste das Stellen verblisterter Medikamente mit Hilfe von Apotheken eine Option sein könnte, die AMTS bei den so betreuten Patienten zu unterstützen.

Nur zu gut ist bekannt, dass mit zunehmendem Lebensalter das Risiko steigt, dass es zu unerwünschten Arzneimittelereignissen (UAE) kommt: Zum einen verändern sich Pharmakokinetik und Pharmakodynamik, zum anderen fördert jedes zusätzlich eingenommene Arzneimittel per se die Gefahr von Interaktionen und UAE. Hinzu kommt: Je mehr Medikamente nötig sind, desto häufiger kommt es zu Fehlern in der Dosierung und Einnahme.

\section{Blick ins Ausland}

In einigen europäischen Ländern ist man bereits weiter, in Deutschland gibt es über
Einzelprojekte hinaus noch keine einheitliche Linie, wie Prof. Stefan Klein von der Universität Münster feststellt. Er verweist auf Länder wie Finnland, Norwegen, Dänemark, Schweden oder die Schweiz, in denen es inzwischen selbstverständlich sei, bei Multimedikation u. a. Verblisterung/ Dispensiervorrichtungen einzusetzen, integriert in ein umfassendes Medikationsmanagement und auf der Grundlage gesetzlicher Regelungen - auch zur Erstattung.

Die Bandbreite organisatorischer Lösungen sei dabei groß, angefangen von nationalen Blisterzentren wie in Finnland, über regionale Blisterzentren wie in Norwegen bis hin zu apothekenbasierten Lösungen wie in der Schweiz.

\section{Deutschland hinkt hinterher}

In Deutschland fehlt es Kleins Ansicht nach hingegen an patienten- und innovationsorientierten Diskursen. Wichtige Fragen wie: Welchen Beitrag kann ein Wochenblister zur Arzneimittelversorgung und AMTS leisten? Wie muss ein Wochenblisterkonzept medizinischpharmazeutisch, technisch und organisatorisch ausgestaltet sein? Wie kann es in das Gesundheitssystem integriert werden? seien zudem noch nicht ausreichend geklärt.

Erste Reaktionen. In der Politik scheint das Thema inzwischen angekommen zu sein. So hat vor kurzem der Pflegeexperte der Unionsfraktion im Bundestag, Erwin Rüddel, mehr Koordination bei der
Medikation durch Arzt und Apotheker stationär wie ambulant - gefordert und in diesem Zusammenhang darauf verwiesen, dass etwa eine passgenaue Verblisterung auch die Pflege entlasten könnte.

\section{Pflegepraxis}

Bereits im Alltag angekommen und integriert ist das Verblistern in der Diakonie Wuppertal. Hier wird nicht nur in stationären Einrichtungen, sondern auch in der ambulanten Pflege diese Versorgungsform erprobt. Wichtig für eine gut funktionierende Zusammenarbeit zwischen Apotheke und Pflegedienst sind die örtliche Nähe mit guter Erreichbarkeit und guten Öffnungszeiten, eine hohe Flexibilität bei der Anlieferung der Medikamente und Blister (nach Dienstschluss und am Wochenende), die Möglichkeit, auch kurzfristig auf Medikationsänderungen zu reagieren, eine hohe Fachkompetenz bei Arzneimittelfragen allgemein, die Option, den Pflegedienst durch Fortbildungen und Beratung zu unterstützen, stete Aktualität der Software, was für AM-Rückfragen wichtig ist, und ein (eventuelles) professionelles softwaregestütztes Medikations- und Rezeptmanagement. Springer-gup/red

\section{SpringerMedizin.at \\ Weitere Informationen unter: \\ www.SpringerMedizin.at/}
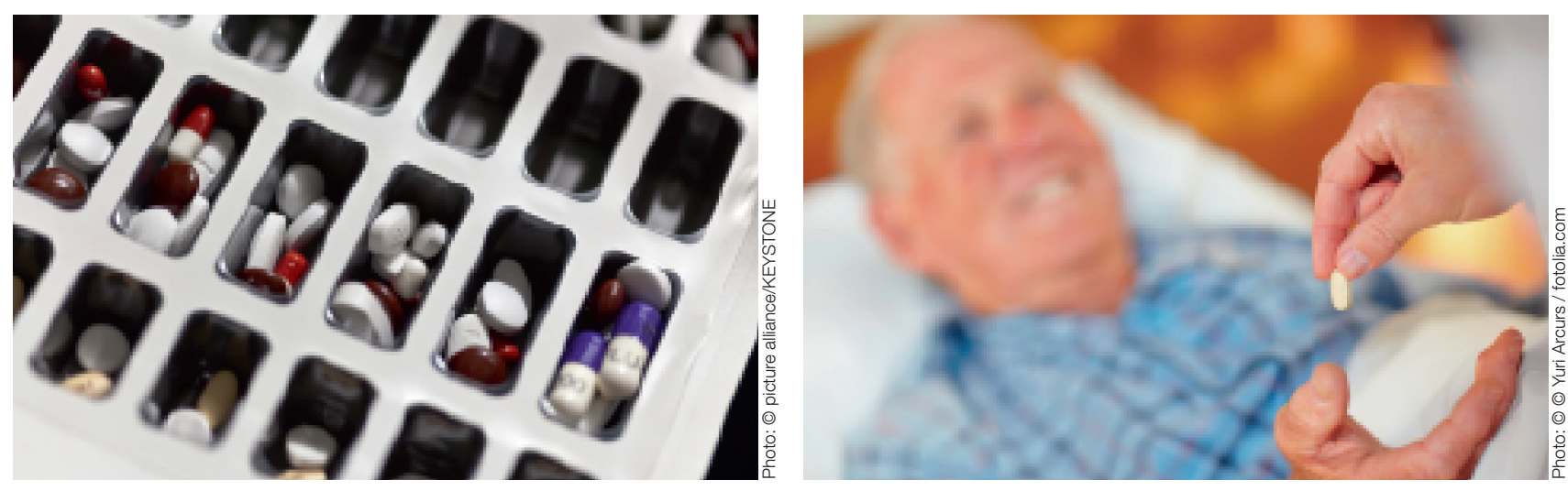